\title{
Majocchi's granuloma: current perspectives
}

This article was published in the following Dove Press journal: Infection and Drug Resistance

\author{
Hazal Boral' \\ Murat Durdu² \\ Macit Ilkit' \\ 'Division of Mycology, Department \\ of Microbiology, Faculty of Medicine, \\ University of Çukurova, Adana, Turkey; \\ ${ }^{2}$ Department of Dermatology, Faculty \\ of Medicine, Bașkent University Adana \\ Hospital, Adana, Turkey
}

Correspondence: Macit llkit

Division of Mycology, Department of Microbiology, Faculty of Medicine, University of Çukurova, Adana 0I330, Turkey

Tel +90532 2860099

$\mathrm{Fax}+903224573072$

Email macitilkit@gmail.com

\begin{abstract}
Majocchi's granuloma (MG) is a rare fungal infection of the dermis that is mainly caused by dermatophytes (in $\geq 95 \%$ of cases); the most frequently identified cause is anthropophilic Trichophyton rubrum. In the rest of the cases, the causes are non-dermatophytic fungi such as Aspergillus species. This review aimed to provide information about the current perspectives on MG regarding its clinical characteristics, predisposing factors, laboratory diagnosis, and treatment strategies. Although the lower extremities were reported to be the most common site of infection, facial involvement has been predominant in the past 5 years. Our literature research showed that the most common predisposing factor (55\%) is the use of topical steroid creams without potassium hydroxide examination during treatment of erythematous squamous dermatoses. A reliable diagnosis of MG is based on histopathological examination, including fungal culture and molecular analyses. MG should be treated not only with topical agents but also with systemic antifungal agents that are continued until the lesions are completely resolved. In systemic treatment, the most preferred drug is terbinafine, because of its efficacy, side effects, and safety.
\end{abstract}

Keywords: dermatomycosis, histopathology, immunosuppression, predisposing factor, Trichophyton rubrum

\section{Introduction}

Dermatophytes are highly specialized keratinophilic and keratinolytic fungi that consist of seven genera, including Trichophyton, Microsporum, Epidermophyton, and the recently introduced Arthroderma, Paraphyton, Nannizzia, and Lophophyton. ${ }^{1}$ Although dermatophytes are the most common human fungal pathogens worldwide, these fungi are neglected because 1 ) they uncommonly cause a life-threatening disease ${ }^{2} 2$ ) in vitro resistance to the first choice of antifungal drugs has been reported, but it is not very common; ${ }^{3}$ and 3 ) most of the effective antifungal drugs are accessible in most countries. ${ }^{4}$ However, besides the ability of these fungi to cause infections in both immunosuppressed and immunocompetent individuals, a growing population of individuals with diabetes and immunosuppression, improvements in medical device technology, and the prolonged life spans of these patients make these fungi more noticeable. ${ }^{5}$

Majocchi's granuloma (MG) is an inflammatory and granulomatous, dermatophytic infection that is classified into two forms, depending on the affected individual's health situation and clinical picture. The first form is mainly observed in healthy individuals and is defined as a perifollicular, papular form induced by penetrating trauma that is mostly observed in the lower extremities. The second form is granulomatous, related 
to immunosuppression, seen in a nodular form, and usually appears on the upper extremities. ${ }^{6}$ The main cause of $\mathrm{MG}$ is Trichophyton rubrum, followed by T. mentagrophytes, $T$. violaceum, and T.tonsurans. However, several fungi, such as T. interdigitale, Microsporum canis, Nannizzia gypsea (former M. gypseum), Epidermophyton floccosum, and Aspergillus species, can also cause MG. ${ }^{7-10}$

Throughout our review of the epidemiological characteristics and treatment strategies of $\mathrm{MG}^{11}$ we noticed that the number of cases of $\mathrm{MG}$ has been rising over the past 5 years. In addition, we are aware that there is some confusion regarding the classification of this invasive infection. Hence, in this review, we aimed to provide up-to-date information about the current knowledge on MG, including demographic characteristics, clinical features, predisposing factors, and diagnostic and treatment strategies for the disease.

\section{Search strategy}

We searched PubMed (MEDLINE) and Google Scholar for MG cases that were published in the English-language literature between August 2011 and November 2017, using the key words "Majocchi's granuloma," "trichophytic granuloma," and "dermatophytic granuloma." Other types of invasive or disseminated dermatophyte infections were excluded from the present review. The clinical and demographic characteristics of 33 patients with MG from 32 articles were evaluated.

\section{Invasive dermatophytosis and MG}

Although dermatophytes require keratin for nutrition, in some circumstances, they can be isolated from the deeper layers of the skin. ${ }^{12-16}$ In our previous review, we classified these infections as follows: 1) MG (nodular, granulomatous perifolliculitis); 2) deeper dermal dermatophytosis; 3) disseminated dermatophytosis; and 4) mycetoma and pseudomycetoma (Figure 1). ${ }^{11}$

Importantly, in the case of dermis invasion by dermatophytes, the immune response determines the clinical picture as follows: 1) a granulomatous inflammation around the hair follicle is called MG. Histopathologically, MG demonstrates a nodular perifollicular granulomatous infiltrate of lymphoid cells, macrophages, epithelioid cells, multinucleated giant cells, and neutrophils. Unlike superficial dermatophytoses, fungal hyphae and spores can be detected not only on the surface of the epidermis but also within or around the hair follicles (Figure 1); ${ }^{16}$ 2) in mycetoma, dermal fungal elements are surrounded by an eosinophilic material, including antigenantibody complexes and debris from host inflammatory cells (Splendore-Hoeppli reaction); 3) dermal invasion and a mild immune response without perifollicular granulomatous inflammation or a Splendore-Hoeppli reaction are called deep dermal dermatophytosis; 4) the clinical picture that consists of vascular involvement and dissemination to other organs is called disseminated dermatophytosis (Figure 1). In this latest form, fungi can be isolated from sputum, blood, or other tissue samples in addition to skin biopsy samples. ${ }^{14,16-18}$

Recently, Rouzaud et a ${ }^{19}$ reported the clinical and histological differences between deep dermatophytosis and MG. However, a patient's immune status, the type and location of the lesion, and direct microscopic examination with potassium hydroxide $(\mathrm{KOH})$ may not be helpful to reliably diagnose $\mathrm{MG}$.

\section{Pathogenesis}

Dermatophytes degrade the keratin in nonliving keratinized tissues to survive. However, in the case of MG, the fungi must survive in the dermal and subcutaneous tissues. Although the underlying mechanisms of the pathogenesis of $\mathrm{MG}$ are not well understood, there are some proposals for this mechanism, and they all rely on several factors that are associated with the host and microorganism.

The first and most important host factor is a physical skin barrier that prevents fungal skin infections. ${ }^{20}$ Physical trauma of the skin due to shaving or scratching and immunosuppression are believed to cause fungal invasion. This invasion occurs because of damage to the epidermal barrier's integrity and follicular disruption; thus, microorganisms, along with keratin and necrotic materials, can enter the dermis. Fungi must hide from the host's immune system, and they cause an inflammatory response during infection. Fungal LysM domain-associated proteins mask chitin on the fungal cell wall and regulate fungal growth and development. ${ }^{21}$ Fungi also have several enzymes, such as lipases, esterases, and collagenases. ${ }^{22}$ Moreover, the microorganisms express several genes that encode the key components of the glyoxylate pathway (i.e. isocitrate lyase and malate synthase) and excrete a large amount of sulfite to degrade the components of the skin. ${ }^{23,24}$

Dermatophytes can cause deep and invasive infections under some acquired or congenital immunosuppressive conditions. For instance, disseminated dermatophytosis might be associated with lymphopenia, reduced complement C3 and C4, and hypogammaglobulinemia. ${ }^{25}$ Additionally, the deficiency of autosomal-recessive caspase recruitment domain-containing protein 9 , which has effects on the signal transducer and activator of the transcription 3 pathway and interleukin (IL)-17 and IL-22 secretion, was also reported in 17 patients with deep dermatophytosis. ${ }^{26-28}$ 
A

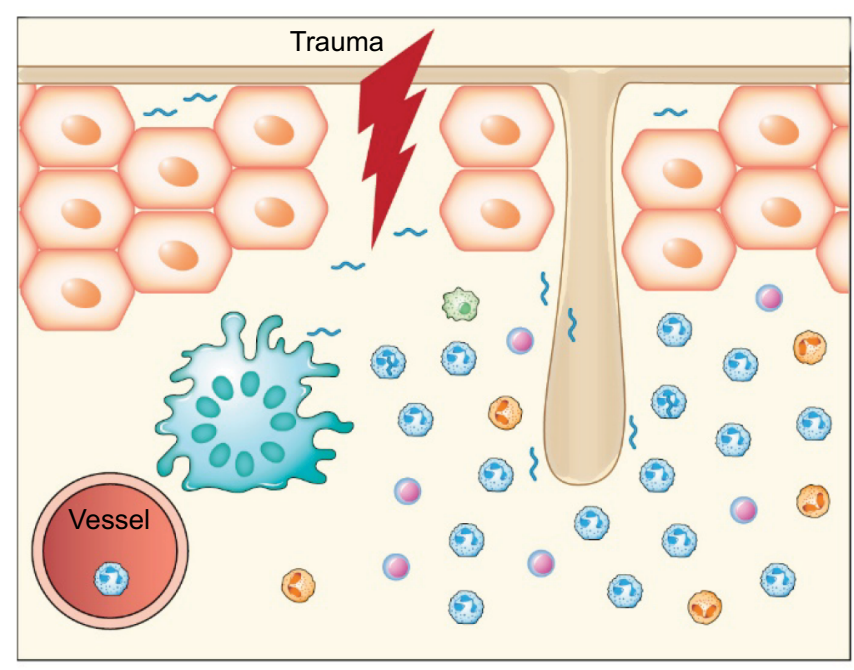

C

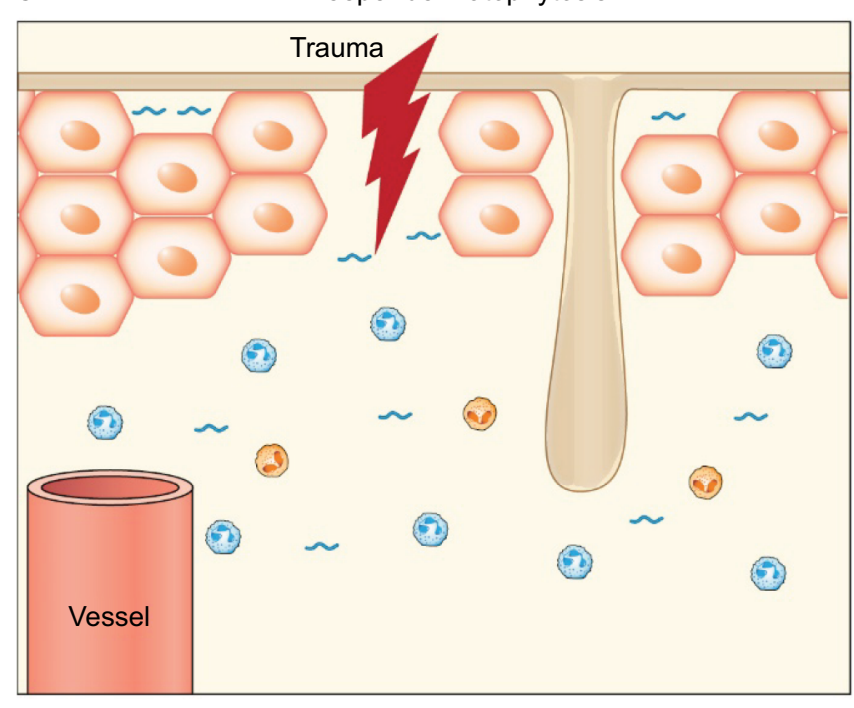

B

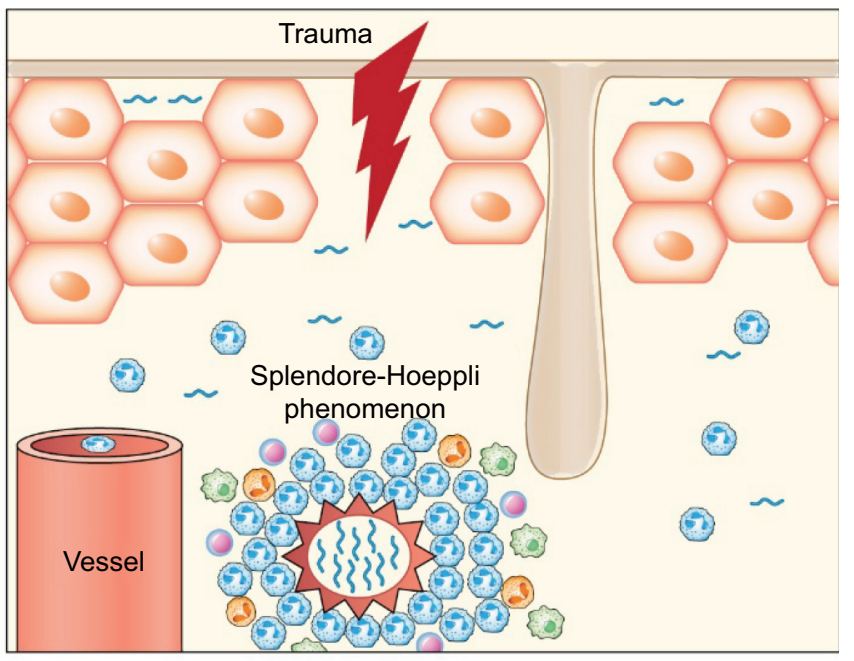

。

Disseminated dermatophytosis

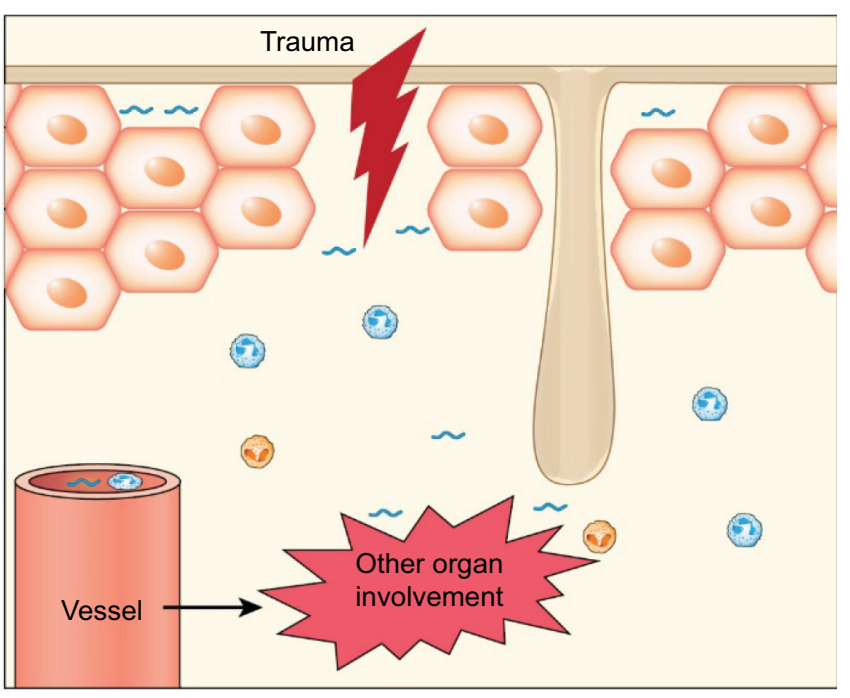

Neutophils

Eosinophils

Lymphocytes

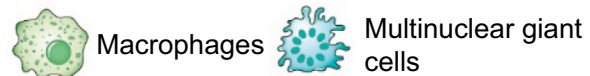

Figure I Pathogenesis of invasive dermatophytosis.

Notes: Physical trauma impairs the epidermal barrier. Penetration of the dermatophytes into the skin causes a granulomatous, inflammatory response, including neutrophils $(\mathrm{N})$, eosinophils (E), lymphocytes (T), macrophages (M), and multinuclear giant cells (MGC). Majocchi's granuloma (A), mycetoma (B), deeper dermatophytosis (C), and disseminated dermatophytosis (D).

Host factors also affect the characteristics of the infection. In a patient with pancytopenia, dermal dermatophytosis without granuloma- or dermatophyte-related sepsis might develop. However, in a patient with partial immunosuppression, granuloma, abscesses, and mycetoma may occur. ${ }^{12,14}$ The host also uses several mechanisms to control the infection. Antimicrobial peptides such as cathelicidins and defensins protect the patient against fungi, and they also promote epidermopoiesis to clear the infection. ${ }^{29,30}$ In addition, natural killer cells, neutrophils, and macrophages also respond to dermatophytosis. Therefore, therapeutic immunosuppression causes lower cellular immunity and ingestion/killing rate of fungal spores. ${ }^{31}$

\section{Source of infection and possible predisposing factors}

The available data in the literature provide some predictions about the source of infection and predisposing factors of 
MG. It commonly occurs in the presence of chronic dermatophytoses, such as tinea unguium and tinea corporis. ${ }^{13,28,32-36}$ These infections may be a source of MG in cases wherein the skin barrier is destroyed. Moreover, shaving the legs or pubic area, sexual contact, and occupation should also be investigated..$^{7,837,38}$ In this review, four of the patients were thought to have been in contact with an animal, suggesting that animal exposure was a predisposing factor of MG. Three of these patients had been in contact with guinea pigs. ${ }^{34,39,40}$ Guinea pigs are often cryptic carriers, ${ }^{41}$ and the clinician should consider the zoophilic characteristics of dermatophytes and whether the patient has a pet or is in frequent contact with animals.

Preexisting dermatophytosis is a major risk factor of MG. Consistently, Kershenovich et $\mathrm{a}^{142}$ reported that the anatomic regions that are involved in preexisting dermatophytosis are the possible origins of MG. This was also evident in our review, which reflected that 10/24 immunocompetent and 7/9 of immunosuppressed patients had prior or concomitant dermatophytic infections. The lesions that were reported in these patients were related to MG, except those in two patients. One was an immunocompetent male patient who had lesions on his suprapubic and inguinal regions and a tinea barbae as a prior dermatophytosis. ${ }^{7}$ However, the patient had a history of unprotected sexual exposures in Thailand. The other was a healthy 58-year-old man who had tinea pedis prior to developing MG lesions on his left forearm, but the source of infection could not be determined because he did not have a history of any local or general immunosuppressive conditions or animal exposure. ${ }^{43}$

Long-term use of steroids, chemotherapy, and antineoplastic therapy or other immunosuppressive conditions may also lead to MG. ${ }^{32,44-47}$ In particular, steroids have been used successfully to treat many lethal diseases, such as pemphigus. However, many cases with steroid use-related sepsis have also been recorded. Steroids affect the functions of macrophages and neutrophils and reduce the Th1-mediated immune response. After steroid use, lesions may become atypical, and complete resolution of fungal infections may be delayed. . $^{36,48}$ Our analyses revealed that the use of steroids $(n=21)$ was the most common underlying condition.

The increase in organ transplantation led to the extensive use of some immunosuppressive drugs such as tumor necrosis factor alpha inhibitors (e.g. adalimumab). Although these drugs can reduce the side effects of steroids, an MG patient who had used adalimumab was reported. ${ }^{49}$ Similarly, $B R A F$ inhibitors (e.g. vemurafenib) are considered promising treatments for aggressive skin tumors; however, they may also facilitate $\mathrm{MG}$ development. ${ }^{32}$
Dermatophytic infections occur frequently in human immunodeficiency virus- or acquired immune deficiency syndrome (AIDS)-positive patients; however, there is no consensus on whether there is any relationship between the lymphocyte count and cutaneous dermatophytosis..$^{50,51}$ When it occurs, dermatophytosis can be either atypical or widespread in these patients. In addition to invasive and chronic, resistant infections, MG can also develop. ${ }^{52}$ Among the cases that were searched for in this review, only one case with $T$. tonsurans had AIDS. ${ }^{53}$ Several other possible predisposing factors, such as chemotherapy $(\mathrm{n}=1)$ and transplantation [solid organ $(n=3)$ and facial tissue allotransplantation $(\mathrm{n}=1)$ ], were also addressed in some studies.

\section{Clinical characteristics}

The clinical pictures of MG in healthy individuals and immunosuppressed patients differ. A perifollicular, papular form that is induced by penetrating trauma is mostly seen in healthy individuals. On the other hand, the granulomatous form is related to immunosuppression and is seen in nodular forms. ${ }^{6}$ In addition to the papular and nodular forms, plaques, patches, and multiple forms, with or without a crust, can also be seen on the lesions (Figure 2A and B).

We reviewed 32 studies including 33 cases (21 men and 12 women) that were published in the English language literature between August 2011 and November 2017. The mean age of the patients was 38 years (range: 3-65 years), and the mean duration of the infections was 9 months (range: 3 days-60 months). The clinical characteristics of patients with MG are shown in Figure 3. The majority of the patients in

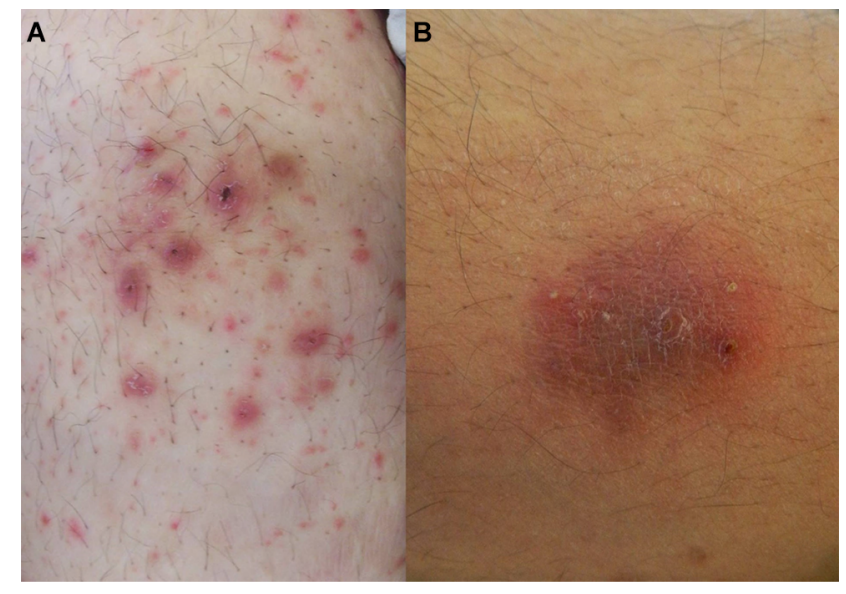

Figure 2 Multiple erythematous papules and nodules with scales and/or crusts are located on the anterior surface of the abdomen in a patient with Majocchi's granuloma (A). Erythematous plaque with pustules, scales, and crusts on the lateral side of the arm in a patient with Majocchi's granuloma (B). 


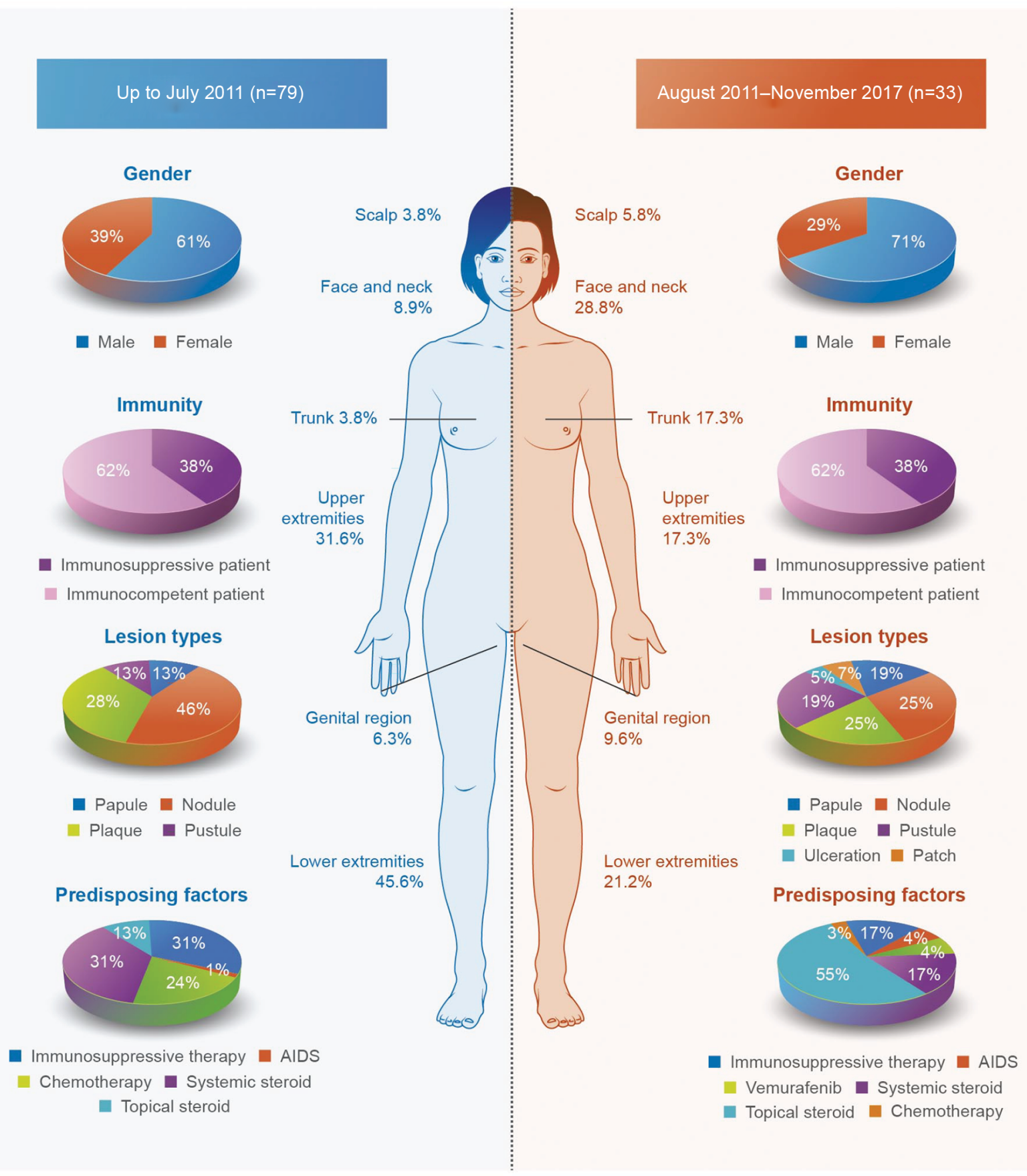

Figure 3 Clinical characteristics of the patients with MG reported in the literature: location of the lesions, sex, immunity, predisposing factors, and type of lesion. Abbreviation: MG, Majocchi's granuloma.

both the immunocompetent and immunosuppressed groups had multiple lesions: $16 / 24$ and $7 / 9$, respectively. The most affected area was the face (37.5\%) among all immunocompetent patients and the lower extremities among the immunosuppressed patients (66.7\%). Although multiple types of lesions $(n=22)$ appeared, the most predominant forms were nodules $(n=19)$ and plaques $(n=19)$. Immunocompetent patients mostly had plaques (62.5\%) and nodules (54.2\%), whereas immunosuppressed patients had nodules (66.7\%) and papules $(55.6 \%)$. In addition, erythroderma and palmoplantar hyperkeratosis have been reported in a patient with AIDS. ${ }^{53}$

The number of reported MG cases has increased remarkably in the past 6 years $(n=33)$, compared to that between 
1883 and $2011(n=79)$. Moreover, the frequency of facial involvement was also prominently higher (36.4\%) than that previously reported $(6.3 \%) .{ }^{11}$

\section{Laboratory diagnosis}

Diagnosing MG requires detection of not only dermatophytes but also perifollicular granulomatous inflammation. The most commonly used method for displaying fungal hyphae and spores is $\mathrm{KOH}$ examination ( $\mathrm{n}=18 ; 7 / 18$ had negative results). However, $\mathrm{KOH}$ examination is insufficient for distinguishing superficial and invasive dermatophytoses. As mentioned previously, perifollicular granulomatous inflammation should be demonstrated for the diagnosis of MG (Figures 4A and B). ${ }^{37}$ However, in this review, we noted that a histopathological examination was not performed in nine patients with MG. Further, in four patients, the staining techniques of histological examination were not mentioned. $7,28,43,46,54$

The stains used in histopathological examinations are very important. Although histopathological examination is the "gold standard" method for demonstrating granulomatous infiltration, hematoxylin-eosin (HE) staining, which is used in routine histopathology, may be insufficient in detecting fungal elements. The most frequently used staining methods for eliminating this deficiency are the periodic acid-Schiff (PAS) $(n=18)$ and Grocott-Gomori's methenamine silver (GMS) $(n=5)$ methods. ${ }^{49,54,55}$

PAS staining is more preferable in the histopathological examination of samples containing suspected fungal infection than GMS because it is easy to perform and has higher sensitivity and negative predictive values (Figure 4C). ${ }^{56}$ When the fungal elements on the suspected samples are numerous, HE staining can also be helpful. However, when there are few fungal elements, they may be overlooked $.^{57} \mathrm{HE}-$ stained preparations can be examined under an immunofluorescence microscope, and fungi are shown as autofluorescent particles (Figure 4D) ${ }^{58}$ On the other hand, GMS staining can be more helpful because it has greater contrast than PAS staining. However, there are no adequate data to conclude that GMS staining is superior to PAS staining. Although GMS staining has an advantage over PAS because it has better powers of detection on low- and intermediate-power microscopy and better contrast to detect fungal elements

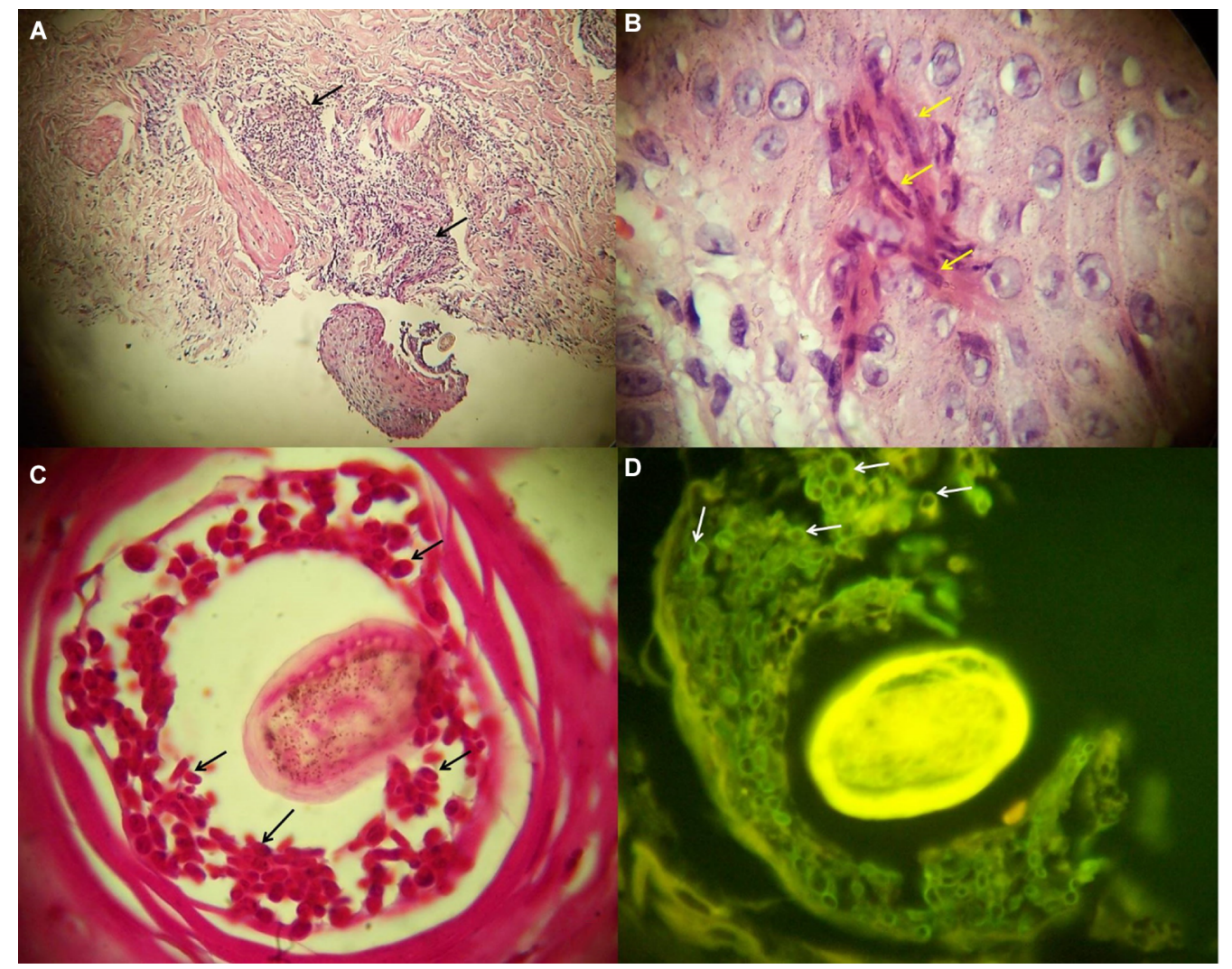

Figure 4 Histopathological findings of a patient with MG.

Notes: (A) The histopathology showed perifollicular, granulomatous inflammation (arrows). (B) Hyphae (arrows) are seen with great magnification. (C) Perifollicular spores (arrows) were positively stained with PAS staining. (D) In the HE-stained slides, spores (arrows) showed autofluorescence under an immunofluorescence microscope. (A, $\mathrm{HE} \times 100$; B, D, HE $\times 1000$; C, PAS $\times 1000$ ).

Abbreviations: MG, Majocchi's granuloma; PAS, periodic acid-Schiff staining; HE, hematoxylin and eosin. 
easily, it is time and temperature dependent, requires an expert technician, and contains hazardous compounds such as chromic acid. ${ }^{56,59,60}$

As histological examination is not sufficient for the identification of a fungus, especially in immunosuppressed patients, it is important to use molecular-based techniques, such as internal transcribed spacer (ITS) sequencing, for identifying fungal species. ${ }^{1,55,61,62} \mathrm{~A}$ fungal culture is required both to detect fungal pathogens and to recognize the species eventually by combining histological analysis and ITS sequencing. Among the studies that were reviewed, fungal culturing was performed for 28 cases, only 1 of which was negative.$^{63}$ In only seven patients, the isolates were identified molecularly using ITS primers. Performing more than one technique, that is, culturing and microscopy, almost always leads to the detection of fungal elements. ${ }^{10,35,53,64-66}$

\section{Etiological agents}

The causes of dermatophytosis depend on the geographic region. Consistently, the etiology of MG may also differ. However, T. rubrum is the most isolated fungal agent of $\mathrm{MG}$ in both immunocompetent and immunosuppressed individuals worldwide. Additionally, T. interdigitale, T. tonsurans, T. violaceum, M. canis, M. ferrugineum, N. gypsea, and E. floccosum were also reported. , $8,10,35,49,66$ In this review, the most common fungal isolate was T. rubrum $(\mathrm{n}=15)$, followed by $T$. mentagrophytes $(\mathrm{n}=5), T$. interdigitale $(\mathrm{n}=2), N$. gypsea $(\mathrm{n}=2), T$. tonsurans $(\mathrm{n}=2)$, and $T$. violaceum $(\mathrm{n}=1)$ (Table 1$)$.

Non-dermatophytic fungi such as those belonging to the genera Phoma and Aspergillus were also reported as etiological agents of MG. ${ }^{9,67}$ Among the 33 cases that were reviewed, only one study reported the presence of a nondermatophytic but keratinophilic fungus, a Malbranchea species, in an immunocompetent patient who had eczema as the underlying disease. ${ }^{68}$

Table I The causative fungi that were isolated from patients with MG

\begin{tabular}{ll}
\hline Causative fungi & Frequency (\%) \\
\hline Dermatophytic fungi & 96.4 \\
Trichophyton rubrum & 55.6 \\
$T$. mentagrophytes & 18.5 \\
$T$. interdigitale & 7.4 \\
$T$. tonsurans & 7.4 \\
$T$. violaceum & 3.7 \\
Nannizzia gypsea & 7.4 \\
Non-dermatophytic fungus & 3.6 \\
Malbranchae sp. & 3.6 \\
\hline
\end{tabular}

Abbreviation: MG, Majocchi's granuloma.

\section{Differential diagnosis}

MG can be confused with diseases that also cause chronic erythematous papules and nodules. Due to the presence of pain in these lesions, they are usually perceived as symptoms of bacterial infections, and this confusion results in patients receiving antibiotic treatment. Other chronic infections (e.g. mycobacterial infections, deep fungal infections, disseminated toxoplasmosis, and cutaneous leishmaniasis) may also be misleading. ${ }^{11}$ In addition to histopathology, bacterial, fungal, and parasitic examinations, as well as polymerase chain reaction and other molecular diagnostic tools, are crucial for reliable organism detection. Notably, when the lesion involves the face, it can imitate granulomatous rosacea and granuloma faciale. Painful nodules also imitate erythema nodosum, thrombophlebitis, and erythema induratum bazin. In immunosuppressed patients, it is important to distinguish MG from some tumoral diseases such as Kaposi sarcoma and lymphoma. ${ }^{36}$

\section{Treatment}

Oral potassium iodide, local X-radiation, and topical 2-dimethylamino-6-( $\beta$-diethylaminoethoxy)-benzothiazole $\left(\right.$ Asterol ${ }^{\circledR}$ ) were used to treat $\mathrm{MG}$ before antifungal treatments were discovered. Antifungal drugs are used topically and/or systemically. Although topical antifungal therapy is usually sufficient for the treatment of superficial dermatophytoses, systemic treatment is also required to treat tinea capitis, onychomycosis, invasive dermatophytoses, and widespread superficial dermatophytoses. The selection of antifungal drug changes with the discovery of novel antifungal drugs. Although ketoconazole was frequently used previously, about half of the patients reported today are treated with terbinafine $(250 \mathrm{mg} /$ day $){ }^{8,13,36,40,43,47,54,68-71}$ Other systemic antifungal drugs are itraconazole (100-200 mg/day), ${ }^{32,44,53,72}$ griseofulvin (250-500 mg/day), ${ }^{10,35,63}$ voriconazole, ${ }^{73}$ and posaconazole. ${ }^{28}$ Antifungal therapy should be continued until the lesions are completely resolved. Depending on the severity of the disease, the duration of MG treatment varies from 1 to 6 months. ${ }^{55,69}$

Rallis et $\mathrm{al}^{37}$ reported a patient who did not respond to systemic itraconazole treatment, but responded well to systemic terbinafine treatment. Liu et $\mathrm{al}^{73}$ reported the case of a patient with a mixed infection of T. rubrum and Klebsiella pneumoniae and treated this case first with systemic antibiotics (combined cefoselis and levofloxacin for 7 days) and then voriconazole ( $200 \mathrm{mg}$, twice daily). Although some newer antifungal drugs were developed after terbinafine, the interaction of novel antifungal drugs is higher than that 
of terbinafine. Post-inflammatory pigmentation, atrophic scarring, and alopecia may develop following the use of antifungal treatment. $7,34,36,37,47,70$

\section{Conclusion}

MG is an uncommon fungal infection that is mostly related to local physical trauma of the skin, followed by disruption of the hair follicles. It may occur in both immunosuppressed and immunocompetent individuals. The source of MG can be a prior dermatophyte infection, exposure to infected or asymptomatic animals or humans, and local or general immunosuppressive conditions.

The diagnosis of MG should be verified by histological examinations, and PAS or GMS staining reveals evidence of the infection. Recognizing the fungal species using conventional and/or molecular methods is also crucial, particularly in immunosuppressed patients. Additionally, understanding the clinical, epidemiological, and histological characteristics of the infection depends on an accurate and reliable clinical and mycological diagnosis. MG can mimic several other infections; therefore, it is important to differentiate $\mathrm{MG}$ and begin treatment as soon as possible. Topical antifungal agents do not respond to treatment, and systemic antifungal agents should be applied at a proper dose and for an appropriate duration. Further studies in this field should focus on proposing a guideline that includes the current diagnostic and management procedures of MG.

\section{Author contributions}

All authors contributed towards data analysis, drafting and critically revising the paper and agree to be accountable for all aspects of the work.

\section{Disclosure}

The authors report no conflicts of interest in this work.

\section{References}

1. de Hoog GS, Dukik K, Monod M, et al. Toward a novel multilocus phylogenetic taxonomy for the dermatophytes. Mycopathologia. 2017;182(1-2):5-31.

2. Bongomin F, Gago S, Oladele RO, Denning DW. Global and multinational prevalence of fungal diseases - estimate precision. J Fungi. 2017;3(4):pii,E57.

3. Yamada T, Maeda M, Alshahni MM, et al. Terbinafine resistance of Trichophyton clinical isolates caused by specific point mutations in the squalene epoxidase gene. Antimicrob Agents Chemother. 2017;61(7):e00115-17.

4. Kneale M, Bartholomew JS, Davies E, Denning DW. Global access to antifungal therapy and its variable cost. J Antimicrob Chemother. 2016;71(12):3599-3606.
5. Scheen A, van Gaal L. Combating the dual burden: therapeutic targeting of common pathways in obesity and type 2 diabetes. Lancet Diabetes Endocrinol. 2014;2(11):911-922.

6. Radentz WH, Yanase DJ. Papular lesions in an immunocompromised patient. Trichophyton rubrum granulomas (Majocchi's granuloma). Arch Dermatol. 1993;129(9):1189-1190, 1192-1193.

7. Gallo JG, Woods M, Graham RM, Jennison AV. A severe transmissible Majocchi's granuloma in an immunocompetent returned traveler. Med Mycol Case Rep. 2017;18:5-7.

8. Parmar NV, Asir GJ, Rudramurthy SM. Atypical presentation of Majocchi's granuloma in an immunocompetent host. Am J Trop Med Hyg. 2017;96(1):1-2

9. Saadat P, Kappel S, Young S, Abrishami M, Vadmal MS. Aspergillus fumigatus Majocchi's granuloma in a patient with acquired immunodeficiency syndrome. Clin Exp Dermatol. 2008;33(4):450-453.

10. Kanaan IC, Santos TB, Kac BK, Souza AM, Cerqueira AM. Majocchi's granuloma - case report. An Bras Dermatol. 2015;90(2):251-253.

11. Ilkit M, Durdu M, Karakaş M. Majocchi's granuloma: a symptom complex caused by fungal pathogens. Med Mycol. 2012;50(5):449-457.

12. Inaoki M, Nishijima $C$, Miyake $M$, et al. Case of dermatophyte abscess caused by Trichophyton rubrum: a case report and review of the literature. Mycoses. 2015;58(5):318-323.

13. Su HA, Sun PL, Sung WW, et al. Deep dermatophytosis caused by zoophilic strain of Trichophyton interdigitale with successful treatment of itraconazole. Mycopathologia. 2017;182(7-8):715-720.

14. Tirado-González M, Ball E, Ruiz A, et al. Disseminated dermatophytic pseudomycetoma caused by Microsporum species. Int J Dermatol. 2012;51(12):1478-1482.

15. Fukushiro R. Dermatophyte abscess [in Japanese]. In: Fukushiro R (ed). Color Atlas of Dermatophytoses. Tokyo: Kanehara Co.;1999:89-95.

16. Marconi VC, Kradin R, Marty FM, Hospenthal DR, Kotton CN. Disseminated dermatophytosis in a patient with hereditary hemochromatosis and hepatic cirrhosis: case report and review of the literature. Med Mycol. 2010;48(3):518-527.

17. Smith KJ, Welsh M, Skelton H. Trichophyton rubrum showing deep dermal invasion directly from the epidermis in immunosuppressed patients. Br J Dermatol. 2001;145(2):344-348.

18. Isa-Isa R, Arenas R, Isa M. Inflammatory tinea capitis: kerion, dermatophytic granuloma, and mycetoma. Clin Dermatol. 2010;28(2): 133-136.

19. Rouzaud C, Chosidow O, Brocard A, et al. Severe dermatophytosis in solid organ transplant recipients: a French retrospective series and literature review. Transpl Infect Dis. 2018;20(1). Epub 2018 Jan 25.

20. Lee WJ, Kim JY, Song CH, et al. Disruption of barrier function in dermatophytosis and pityriasis versicolor. J Dermatol. 2011;38(11): 1049-1053.

21. Martinez DA, Oliver BG, GräserY, et al. Comparative genome analysis of Trichophyton rubrum and related dermatophytes reveals candidate genes involved in infection. mBio 2012;3(5):e00259-12.

22. Boral H, Metin B, Döğen A, Seyedmousavi S, Ilkit M. Overview of selected virulence attributes in Aspergillus fumigatus, Candida albicans, Cryptococcus neoformans, Trichophyton rubrum, and Exophiala dermatitidis. Fungal Genet Biol. 2018;111:92-107.

23. Monod M. Secreted proteases from dermatophytes. Mycopathologia. 2008;166(5-6):285-294.

24. Zaugg C, Monod M, Weber J, et al. Gene expression profiling in the human pathogenic dermatophyte Trichophyton rubrum during growth on proteins. Eukaryot Cell. 2009;8(2):241-250.

25. Abdel-Rahman SM. Genetic predictors of susceptibility to dermatophytoses. Mycopathologia. 2017;182(1-2):67-76.

26. Puel A, Picard C, Cypowyj S, Lilic D, Abel L, Casanova JL. Inborn errors of mucocutaneous immunity to Candida albicans in humans: a role for IL-17 cytokines? Curr Opin Immunol. 2010;22(4):467-474.

27. Alves de Medeiros AK, Lodewick E, Bogaert DJ, et al. Chronic and invasive fungal infections in a family with CARD9 deficiency. $J$ Clin Immunol. 2016;36(3):204-209. 
28. Lanternier F, Pathan S, Vincent QB, et al. Deep dermatophytosis and inherited CARD9 deficiency. NEngl J Med. 2013;369(18):1704-1714.

29. Matejuk A, Leng Q, Begum MD, et al. Peptide-based antifungal therapies against emerging infections. Drugs Future. 2010;35(3):197.

30. van Kilsdonk JWJ, Jansen PAM, van den Bogaard EH, et al. The effects of human beta-defensins on skin cells in vitro. Dermatology. 2017;233(2-3):155-163.

31. Azad A, Kaufman M, Jayarajan J. Dexamethasone-induced flares of Trichophyton rubrum masquerading as docetaxel cutaneous toxicity: a case report. Cases J. 2009;2:8552.

32. Anforth R, Carlos G, Eiris N, Clements A, Fernandez-Peñas P. Tinea hidden by a vemurafenib-induced phototoxic reaction in a patien with metastatic melanoma taking dexamethasone. Med J Aust. 2015; 203(1):41-42.

33. Rãducan A, Constantin MM. To be or not to be tinea - Misdiagnosis and inappropriate treatment. Dermatovenerol (Buc.). 2017;62:29-32.

34. Trocoli Drakensjö I, Vassilaki I, Bradley M. Majocchi’s granuloma caused by Trichophyton mentagrophytes in 2 immunocompeten patients. Actas Dermosifiliogr (English Edition). 2017;108(1):e6-e8.

35. Kranseler J, Gupta D. Erythematous annular plaques on a 9-year-old's arms and chest. Clin Pediatr. 2018;57(4):488-490.

36. Kim JE, Won CH, Chang S, Lee MW, Choi JH, Moon KC. Majocchi's granuloma mimicking Kaposi sarcoma in a heart transplant patient. J Dermatol. 2011;38(9):927-929.

37. Rallis E, Katoulis A, Rigopoulos D. Pubic Majocchi's granuloma unresponsive to itraconazole successfully treated with oral terbinafine. Skin Appendage Disord. 2015;1(3):111-113.

38. Molina-Leyva A, Perez-Parra S, Garcia-Garcia F. Case for diagnosis. An Bras Dermatol. 2014;89(5):839-840.

39. Liu C, Landeck L, Cai SQ, Zheng M. Majocchi's granuloma over the face. Indian J Dermatol Venereol Leprol. 2012;78(1):113-114.

40. Wargo JJ, Berg A, Gudjonsson JE. Ongoing pleuritic chest pain and a guinea pig: missed pulmonary embolism and Majocchi's granuloma. Am J Med. 2016;129(9):e165-167.

41. Kraemer A, Hein J, Heusinger A, Mueller RS. Clinical signs, therapy, and zoonotic risk of pet guinea pigs. Mycoses. 2013;56(2):168-172.

42. Kershenovich R, Sherman S, Reiter O, et al. A unique clinicopathological manifestation of fungal infection: A case series of deep dermatophytosis in immunosuppressed patients. Am J Clin Dermatol. 2017;18(5):697-704

43. Roy SF, Davar S. Majocchi's granuloma in a 58-year-old man with treatment-resistant annular scaling plaque. CMAJ. 2017;189(48):e1493.

44. Li FQ, Lv S, Xia JX. Majocchi's granuloma after topical corticosteroids therapy. Case Rep Dermatol Med. 2014;2014:507176.

45. Lourdes LS, Mitchell CL, Glavin FL, Schain DC, Kaye FJ. Recurrent dermatophytosis (Majocchi's granuloma) associated with chemotherapyinduced neutropenia. J Clin Oncol. 2014;32(27):e92-e94.

46. Steiner UC, Trüeb RM, Schad K, et al. Trichophyton rubrum-induced Majocchi's granuloma in a heart transplant recipient. A therapeutic challenge. JAAD Case Rep. 2012;6(3):70-72.

47. Zhou S, Yang Y, Liu K, Huang S, Hu Y, Zhou X. A case report of widespread Majocchi's granuloma in a patient with systemic lupus erythematosus. Mycopathologia. 2017;182(5-6):577-581.

48. Gupta V, Bhatia R, Sondhi P, Mahajan R. 'Ring-within-a-ring' appearance: morphological clue to topical steroid abuse in dermatophytosis. J Eur Acad Dermatol Venereol. 2017;31(1):e2-e3.

49. Chou WY, Hsu CJ. A case report of Majocchi's granuloma associated with combined therapy of topical steroids and adalimumab. Medicine. 2016;95(2):e2245.

50. Johnson RA. Dermatophyte infections in human immune deficiency virus (HIV) disease. J Am Acad Dermatol. 2000;43(Suppl 5):S135-142.

51. Rodwell GE, Bayles CL, Towersey L, Aly R. The prevalence of dermatophyte infection in patients infected with human immunodeficiency virus. Int J Dermatol. 2008;47(4):339-343.
52. Ramos-e-Silva M, Lima CM, Schechtman RC, Trope BM, Carneiro S. Superficial mycoses in immunodepressed patients (AIDS). Clin Dermatol. 2010;28(2):217-225.

53. Lyra MR, Muniz Álvarez B, Lanziano AL, et al. Exfoliative erythroderma and palmoplantar hyperkeratosis associated with Majocchi's granuloma by Trichophyton tonsurans in a patient with AIDS. Rev Iberoam Micol. 2017;34(3):185-188.

54. Bakardzhiev I, Chokoeva A, Tchernev G, Wollina U, Lotti T. Tinea profunda of the genital area. Successful treatment of a rare skin disease. Dermatol Ther. 2016;29(3):181-183.

55. Su H, Li L, Cheng B, et al. Trichophyton rubrum infection characterized by Majocchi's granuloma and deeper dermatophytosis: case report and review of published literature. Mycopathologia. 2017;182(5-6):549-554.

56. Reza Kermanshahi T, Rhatigan R. Comparison between PAS and GMS stains for the diagnosis of onychomycosis. J Cutan Pathol. 2010;37(10):1041-1044.

57. Al-Amiri A, Chatrath V, Bhawan J, Stefanato CM. The periodic acidSchiff stain in diagnosing tinea: should it be used routinely in inflammatory skin diseases? J Cutan Pathol. 2003;30(10):611-615.

58. Elston DM. Fluorescence of fungi in superficial and deep fungal infections. BMC Microbiol. 2001;1:21.

59. Shiogama K, Kitazawa K, Mizutani Y, Onouchi T, Inada KI, Tsutsumi Y. New Grocott stain without using chromic acid. Acta Histochem Cytochem. 2015;48(1):9-14.

60. D'Hue Z, Perkins SM, Billings SD. GMS is superior to PAS for diagnosis of onychomycosis. J Cutan Pathol. 2008;35(8):745-747.

61. Mirhendi H, Makimura K, de Hoog GS, et al. Translation elongation factor 1- $\alpha$ gene as a potential taxonomic and identification marker in dermatophytes. Med Mycol. 2015;53(3):215-224.

62. Rezaei-Matehkolaei A, Mirhendi H, Makimura K, et al. Nucleotide sequence analysis of beta tubulin gene in a wide range of dermatophytes. Med Mycol. 2014;52(7):674-688.

63. Lewkowicz C, Lakhiani C. Girl's papular rash will not respond to treatment. Int J Contemp Pediatr. 2017;34(9):42-44.

64. Pietrzak A, Tomasiewicz K, Kanitakis J, et al. Trichophyton mentagrophytes-associated Majocchi's granuloma treated with cryotherapy. Folia Histochem Cytobiol. 2012;50(3):486-489.

65. Bjekić M, Basara JG. Majocchi’s granuloma in a healthy adult man - a case report. Serb J Dermatol Venerol. 2015;7(1):34-40.

66. Khanna U, Dhali TK, D'Souza P, Chowdhary S. Majocchi's granuloma of the breast: a rare clinical entity. Actas Dermosifiliogr (English Edition). 2016;107(7):610-612.

67. Sequeira M, Burdick AE, Elgart GW, Berman B. New-onset Majocchi's granuloma in two kidney transplant recipients under tacrolimus treatment. J Am Acad Dermatol. 1998;38(3):486- 488.

68. Govind A, L'Etoile N, Vasquez G. The first reported case of Majocchi's granuloma with Malbranchea sp. in an immunocompetent patient. Case Rep Infect Dis. 2017;2017:9196528.

69. Bressan AL, Silva RSD, Fonseca JCM, Alves MDFGS. Majocchi's granuloma. An Bras Dermatol. 2011;86(4):797-798.

70. Knoll BM, Hammond SP, Koo S, et al. Infections following facial composite tissue allotransplantation - single center experience and review of the literature. Am J Transplant. 2013;13(3):770-779.

71. Wang T, Xu C, Zhou X, et al. Homozygous ALOXE3 nonsense variant identified in a patient with non-bullous congenital ichthyosiform erythroderma complicated by superimposed bullous Majocchi's granuloma: the consequences of skin barrier dysfunction. Int $\mathrm{J} \mathrm{Mol}$ Sci. 2015;16(9):21791-21801

72. Scott-Lang V, Tidman M. Majocchi's granuloma in an immunocompetent child. Arch Dis Child. 2012;97(Suppl 1):A42.

73. Liu HB, Liu F, Kong QT, et al. Successful treatment of refractory Majocchi's granuloma with voriconazole and review of published literature. Mycopathologia. 2015;180(3-4):237-243. 


\section{Publish your work in this journal}

Infection and Drug Resistance is an international, peer-reviewed openaccess journal that focuses on the optimal treatment of infection (bacterial, fungal and viral) and the development and institution of preventive strategies to minimize the development and spread of resistance. The journal is specifically concerned with the epidemiology of antibiotic resistance and the mechanisms of resistance development and diffusion in both hospitals and the community. The manuscript management system is completely online and includes a very quick and fair peerreview system, which is all easy to use. Visit http://www.dovepress.com/ testimonials.php to read real quotes from published authors.

Submit your manuscript here: https://www.dovepress.com/infection-and-drug-resistance-journal 